\title{
Characterization of Ag nanoparticles biosynthesized by Bacillus sp. HAI4 in different conditions and their antibacterial effects
}

\author{
Mojtaba Taran, Maryam Rad, Mehran Alavi* \\ Department of Biology, Faculty of Science, Razi University P.O. Box, 6714967346, Kermanshah, Iran.
}

\begin{tabular}{l} 
ARTICLE INFO \\
\hline Article history: \\
Received on: 03/06/2016 \\
Revised on: 17/07/2016 \\
Accepted on: 07/08/2016 \\
Available online: 29/11/2016 \\
\hline Key words: \\
Ag nanoparticles, Bacillus \\
sp. HAI4, Qualitek-4 \\
software, UV spectroscopy, \\
SEM, Staphylococcus \\
aureus.
\end{tabular}

\begin{abstract}
In the present study, Ag nanoparticles (NPs) were synthesized by eco-friendly method. Design and analysis of Taguchi experiments was done by the Qualitek-4 software. Effect of $\mathrm{AgNO}_{3}$ concentration $(0.1,0.01$ and 0.001 M), incubation and culturing time (48, 72, 96 hours) as three different levels were measured in NPs biosynthesis. We carried out biosynthesis of NPs through cell-free culture supernatant of Bacillus sp. HAI4. Xray diffraction (XRD), Fourier Transform Infra-Red spectroscopy (FT-IR), Ultraviolet-Visible spectroscopy (UV) and Field Emission Scanning Electron Microscope (FESEM) techniques were applied for NP characterization. Results of Taguchi analysis showed optimum condition with $12.641 \%$ amount which is good result of biosynthesis by this bacterium. XRD analysis proved the crystalline and amorphous nature of synthesized silver NPs. Analysis of FESEM demonstrated that Ag nanoparticles were formed as spherical shapes with size of 33/264 nm. Also, for evaluating of antibacterial effects, maximum zone of inhibition, two important multidrug resistant pathogenesis bacteria, Escherichia coli ATCC 25922 and Staphylococcus aureus ATCC 43300 were used. Three levels of Ag NPs concentrations had antibacterial effect on these bacteria (1, 1 and $0.5 \mathrm{~cm}$ for E.coli and $1,1.1$ and $1.1 \mathrm{~cm}$ for S.aureus), but there was no significant difference between three levels.
\end{abstract}

\section{INTRODUCTION}

Improving disinfection methods in order to fight against pathogenesis microorganisms specifically multidrug resistant bacteria are important in hospital infections (Varshney et al., 2012). For many years, silver ions were common antibacterial that applied in catheters (Jain and Pradeep, 2005). In this case, with knowledge of bactericidal effect on E. coli and Bacillus subtilis, copper and silver ions have been used as antibacterial agents in wastewater of hospitals (Lin et al., 1996; Lin et al., 1998; Blanc et al., 2005; Yoon et al., 2007).

These applications of ions have major disadvantages. As, in these wastewater, silver and copper ions can be remained and act as harmful agents for human and environment health (Blanc et al., 2005).

\footnotetext{
* Corresponding Author

Mehran Alavi, Department of Biology, Faculty of Science, Razi University P.O. Box, 6714967346, Kermanshah, Iran.

Email: mehranbio83 @ gmail.com
}

Nanotechnology and nanoscience are improving these activities of metals nanoparticles (NPs) through unique properties at nanometer scales (10-500 nm, seldom more than $700 \mathrm{~nm}$ ). These new functions of metal NPs are related to several properties such as surface area to volume ratio which are higher than conventional ion forms of metals (Vicky et al., 2010).

Also, NPs have other applications as drug carriers, catalysis and sensors, etc. (Vaseashta and Dimova-Malinovska, 2005).

Nanoparticles synthesis can be obtained through physicochemical and biological techniques. Chemical synthesis methods have the harmful effect on the environment because using hazardous chemical substances (Monika et al., 2015). In contrast to physicochemical methods, biological syntheses have several advantages such as eco-friendly effects. Bacteria, yeast, algae, fungi, higher angiospermic plants and weeds are used for nanoparticles fabrication (Varshney et al., 2012). Among these biological methods, bacteria have been more considerable because its simplicity and pure production (Vidhya et al., 2014). 
Extracellular and intracellular ways can occur in metal NPs synthesis bay bacteria (Kalishwaralal et al., 2010; Saifuddin et al., 2012). Further treatments for release the metal NPs are requiring in intracellular NPs synthesis such as ultrasound and centrifugation (Ganesh Babu and Gunasekaran, 2009). In contrast, simplicity, cheap and large-scale production properties are related to extracellular NPs biosynthesis. Therefore, in experimental studies, extracellular NPs synthesis has been more attention than intracellular one (Vidhya et al., 2014). In this method, Ag NPs can be formed within few minutes from $\mathrm{Ag}+$ ions in the culture supernatant of Escherichia coli ATCC 8739, Bacillus subtillis ATCC 6633, Streptococcus thermophiles ESh1(Abd et al., 2011) and Streptomyces sp. ERI-3(Faghri Zonooz and Salouti, 2011).

In this study, selected strain Bacillus sp. HAI4. was applied to reduction of $\mathrm{Ag}^{+}$ion and conversion it to $\mathrm{Ag}$ nanoparticles. UV-visible spectroscopy, X-ray diffraction (XRD), Fourier Transform Infra-Red spectroscopy (FT-IR), and Scanning Electron Microscopy (SEM) techniques were applied for NP characterization. Also, antibacterial effects of the final reaction were measured on two pathogenesis bacteria strain with multidrug resistant property, Escherichia coli ATCC 25922 and Staphylococcus aureus ATCC 43300.

\section{MATERIAL AND METHODS}

\section{Taguchi methodology experimental design}

In order to optimization of experimental conditions, all the combination experiments were conducted using the assigned parameter values. The Qualitek-4 software was utilized to design and analysis of Taguchi experiments (Taran et al., 2015). Table 1 demonstrates variable factors and their levels in this experiment design.

\section{Microorganism in Ag NPs biosynthesis and preparation of supernatant}

Bacteria Bacillus sp. HAI4 was obtained from bacterial archive, Razi University, Kermanshah. Growth conditions were simple, growth on $0.5 \mathrm{NB}$ (nutrient broth) agar plate. The obtained biomass was washed with phosphate buffer $(\mathrm{pH}$ 7.0) thrice and collected in a 500-ml Erlenmeyer flask. 1M solution of $\mathrm{AgNO}_{3}$ was prepared using de-ionized water, and $100 \mathrm{ml}$ of the solution was added to the biomass harvested at each point of time. The Erlenmeyer flasks were incubated at $37{ }^{\circ} \mathrm{C}$ under agitation (200 rpm) for $24 \mathrm{~h}$.

\section{Characterization}

In order to determination of structure, morphology and elemental composition, the prepared annealed samples were analyzed by X-ray diffraction analysis (XRD), scanning electron micrograph (SEM). Crystallographic study was carried out using EQUNIOX 3000, diffractometer in the scanning range of $20^{\circ}-70^{\circ}$ (20) using $\mathrm{Cu} \mathrm{Ka}$ radiations of wavelength $1.5406 \AA$. Model XL30, Philips, Eindhoven, Field Emission Scanning Electron Microscope (FESEM) was used to study the morphology of the nanoparticles and the elemental analysis. The intensity of absorption peaks and peak absorbance of NPs was examined by UV-Vis spectrophotometer (Tomas, UV 331) from 300 to $800 \mathrm{~nm}$. FT-IR measurements were done by spectrophotometer (Germany, Bruker, Model:ALPHA).

\section{Antibacterial effects}

Escherichia coli ATCC 25922 and Staphylococcus aureus ATCC 45500 as pathogen bacteria were utilized for measurement the effect of antibacterial properties of AgNPs by well diffusion modified ager. Clinical pathogen bacteria were cultivated in Muller Hinton agar (MHA) plates; $5 \mathrm{~mm}$ diameter disks were prepared with the help of a sterilized steel cork borer. Afterwards, different concentrations of AgNPs (0.1, 0.01 and $0.001 \mathrm{M}$ ) were loaded in different disks and after placing the disks on agar the plates were incubated at $37^{\circ} \mathrm{C}$ for $48 \mathrm{hr}$ (Ramyadevi et al., 2012).

\section{RESULTS and DISCUSSION}

The formation of silver nanoparticles was carried out by the culture supernatants of Bacillus sp. HAI4. In this case, bacterial strains were incubated for reduction of silver ion to silver NPs as there was rapid reaction (rapid color change at 10 minutes). Figure 1 shows appearance of a yellowish-brown color in the reaction vessels which suggested the formation of colloidal $\mathrm{Ag}$ NPs. Excitation of surface plasmon vibration may be cause of this color change (Shahverdi et al., 2007). Silver NPs synthesis within hours (Fusarium oxysporum) and minutes (Aspergillus fumigatus) were reported (Bhainsa and D'Souza, 2006). Intensity of color increased after $24 \mathrm{~h}$ of incubation that resulted from high nanoparticles formation.

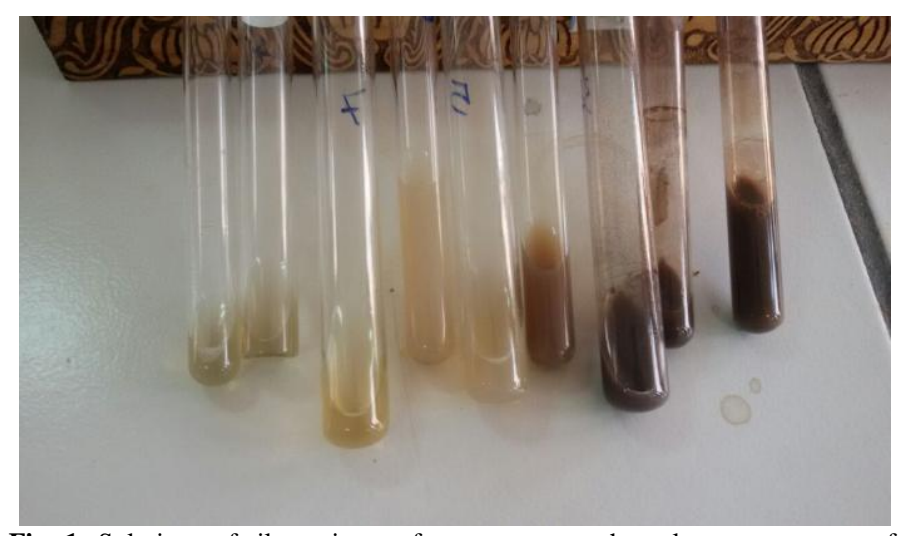

Fig. 1: Solutions of silver nitrate after exposure to the culture supernatant of Bacillus sp. HAI4 (from left to right 0.001, 0.01 and 0.1M ).

The UV-Vis absorption spectra of the supernatant evaluated in the range of $300-800 \mathrm{~nm}$ by a double beam UV-Vis spectroscopy. Figures 2 show a strong broad absorption band located between 340 and $350 \mathrm{~nm}$ for silver NPs prepared by this bacterium. The absorbance in the range of 340-350 nm increases by increasing of $\mathrm{AgNO}_{3}$ concentration. 

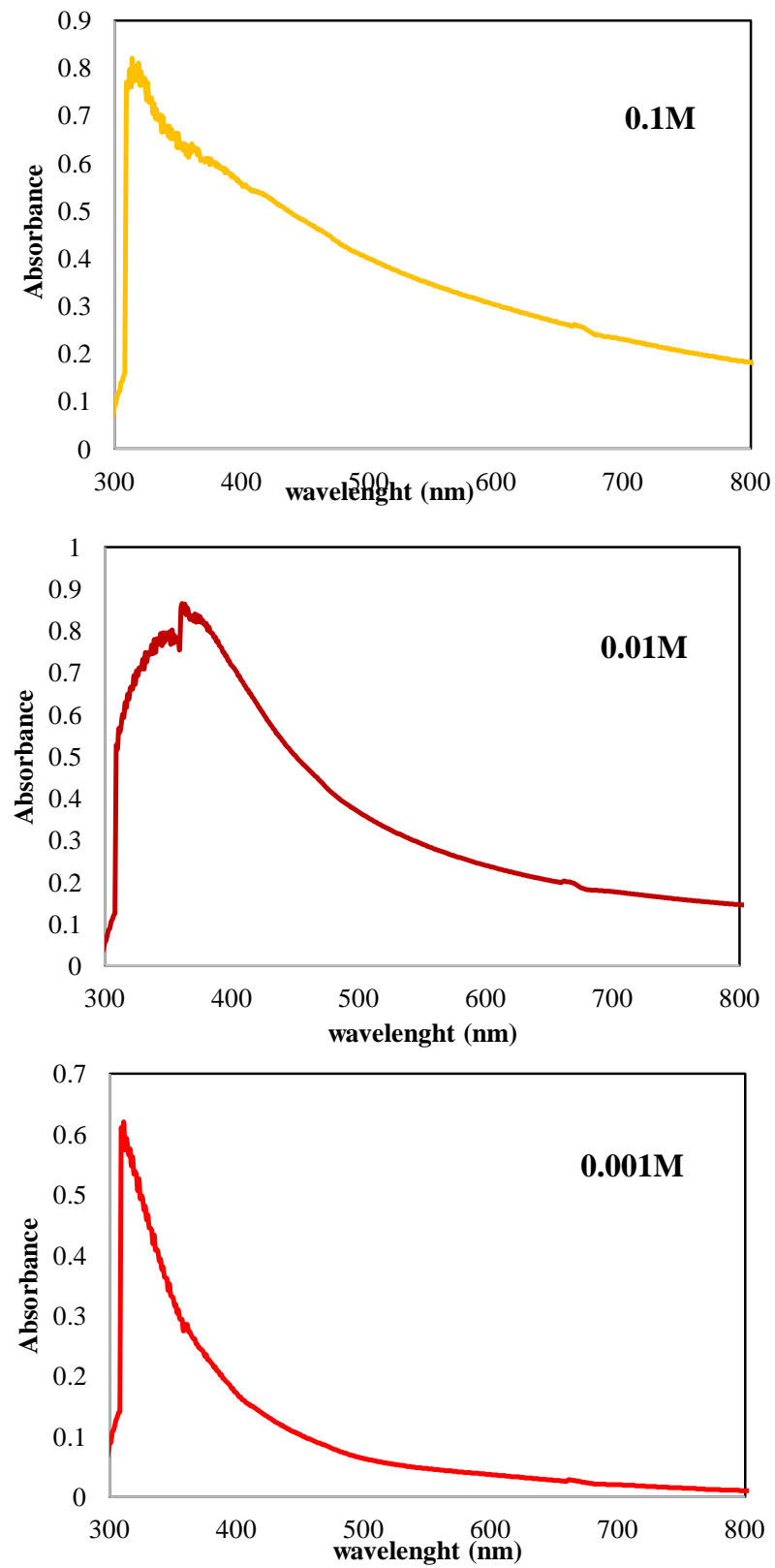

Fig. 2: UV-Vis spectrum of Ag NPs produced by Bacillus sp. HAI4 in three level of concentration $0.1,0.01$ and $0.001 \mathrm{M}$.

Table 1 illustrates effects of three different factors $\left(\mathrm{AgNO}_{3}\right.$ concentration, incubation time and culture time) on the silver NPs biosynthesis by Bacillus sp. HAI4. $\mathrm{AgNO}_{3}$ concentration in level 1(11.326), incubation time in level 2 (4.695), culturing time in level 2 (4.172) had higher effect on the Ag NPs biosynthesis (Fig. 3).

Table 1: Effects of three different factors on the AgNPs biosynthesis.

\begin{tabular}{lccc}
\multicolumn{1}{c}{ Factors } & $\begin{array}{c}\text { Level } \\
\text { 1 (value) }\end{array}$ & $\begin{array}{c}\text { Level } \\
\text { 2 (value) }\end{array}$ & $\begin{array}{c}\text { Level } \\
\text { 3 (value) }\end{array}$ \\
\hline AgNO $_{3}$ concentration & 11.326 & 0.793 & 0.361 \\
Incubation time & 3.457 & 4.695 & 4.327 \\
Culturing time & 3.37 & 4.94 & 4.172 \\
\hline
\end{tabular}
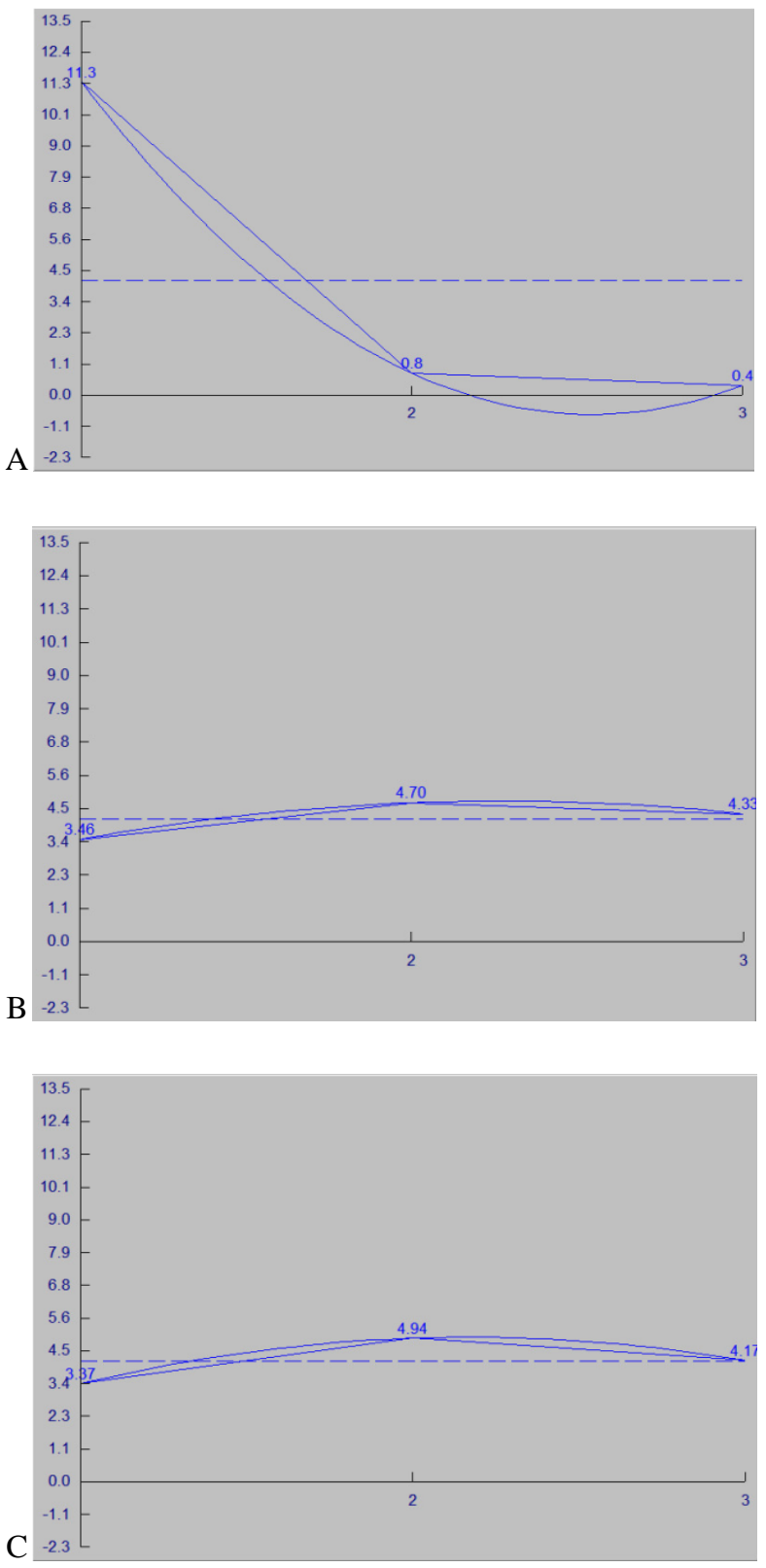

Fig. 3: Taguchi results of average effect of $\mathrm{AgNO}_{3}$ concentration (a), incubation time (b) and culture time (c).

Effective factors in silver nanoparticles synthesis by Bacillus sp. HAI4 are demonstrated by variance analysis. Final column determines each factors which major factor is $\mathrm{AgNO}_{3}$ concentration.

Table 2: Analysis of variance for Ag NPs biosynthesis.

\begin{tabular}{|c|c|c|c|c|c|c|}
\hline Factors & $\underset{8}{8}$ & 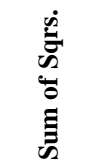 & 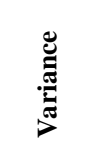 & 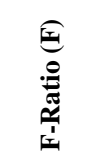 & 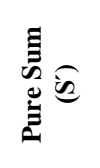 & 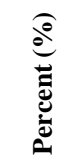 \\
\hline $\begin{array}{l}\mathrm{AgNO}_{3} \\
\text { concentration }\end{array}$ & 2 & 231.354 & 115.677 & 123.672 & 229.483 & 95.875 \\
\hline Incubation time & 2 & 2.433 & 1.216 & 1.3 & 0.562 & 0.235 \\
\hline Culturing time & 2 & 3.697 & 1.848 & 1.976 & 1.827 & 0.763 \\
\hline
\end{tabular}


Table 3 shows optimum conditions for biosynthesis of Ag NPs affected by three factors. Expected result at optimum condition was $12.641 \%$ which is good result of biosynthesis by this bacterium.

Table 3: Optimum conditions of Ag NPs biosynthesis by bacterium.

\begin{tabular}{lcc}
\hline Factors & Level & Contribution \\
\hline $\mathrm{AgNO}_{3}$ concentration & 2 & 7.165 \\
Incubation time & 2 & 0.537 \\
Culturing time & 3 & 0.779 \\
Total contribution from all factors & - & 8.48 \\
Current grand average of performance & - & 4.16 \\
Expected result at optimum condition & - & 12.641 \\
\hline
\end{tabular}

\section{X-ray diffraction analysis}

To confirm the crystalline nature of Ag NPs, further studies were done by X-ray diffraction (Fig. 4). This figure illustrates peaks at $2 \theta$ values of $38 / 09^{\circ}, 46 / 09^{\circ}, 64 / 52^{\circ}, 77 / 51^{\circ}$ corresponding to XRD planes (111), (200), (220) and (311) Bragg's reflection based on the fcc structure of Ag NPs. Also, a few unmarked peaks were noticed in the vicinity of the characteristic peaks. These peaks can be resulted from the existing of capping agent stabilizing the NPs. The sizes of gained nanoparticles were estimated to be in the range of $33 / 264 \mathrm{~nm}$ using Debye-Scherer eq. (1):

$\mathrm{d}=\mathrm{k} \lambda /(\beta \cos \theta)$

where $\mathrm{K}$, known as Scherer's constant, ranges from 0.9 to $1.0, \lambda$ is $1.5418 \AA$, which is the wavelength of the $\mathrm{X}$-Ray radiation source, $\beta 1 / 2$ is the width of the XRD peak at half height and $\theta$ is the Bragg angle (Fig. 4). XRD analysis showed the crystalline and amorphous nature of synthesized silver NPs. Analysis of SEM demonstrated that Ag nanoparticles were formed as aggregates and had spherical shapes (Fig. 5).

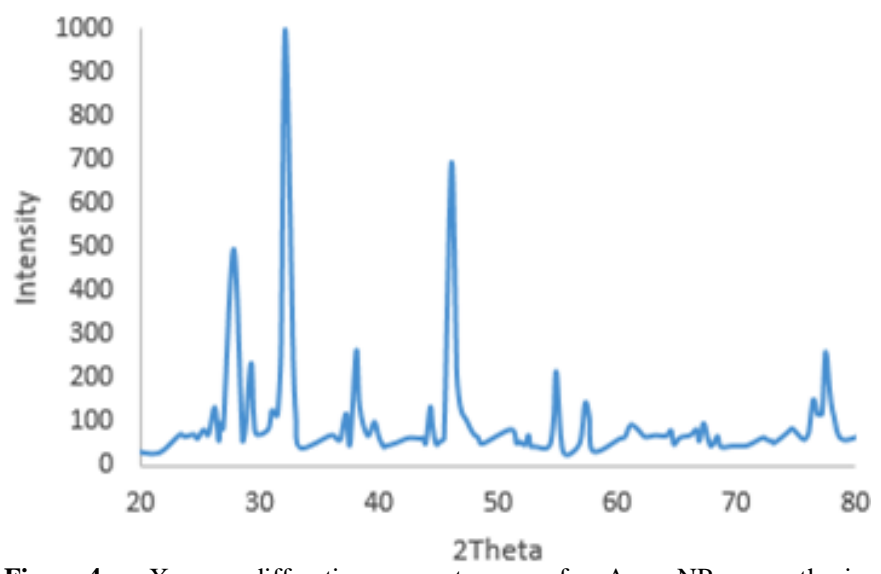

Fig. 4: X-ray diffraction spectrum of Ag NPs synthesized from $0.1 \mathrm{M} \mathrm{AgNO}_{3}$ treated Bacillus sp. HAI4 cell free supernatant at $28^{\circ} \mathrm{C}$.
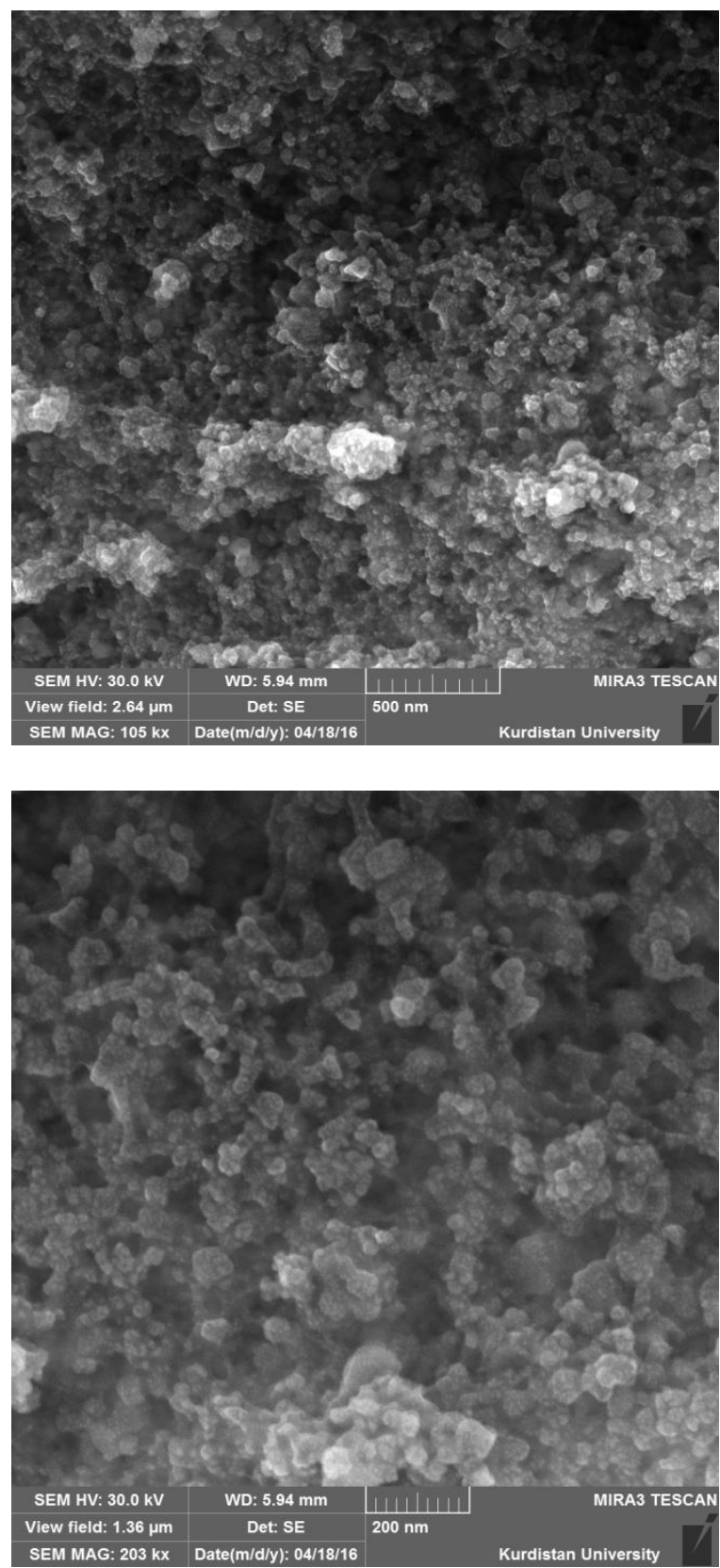

Fig. 5: Scanning electron microscopy (SEM) image of AgNPs produced by culture supernatant of Bacillus sp. HAI4.

\section{FTIR analysis}

This analysis demonstrates synthesized silver nanoparticles peaks at $834 ; 1,379 ; 1,634 ; 2,432 ; 2,972 ; 3,430 \mathrm{~cm}^{-1}$. Three considerable bands could be observed from the figure 6 . The intense peaks at 1379 and $1634 \mathrm{~cm}^{-1}$ corresponds to alkyl and $\mathrm{C}=\mathrm{C}$ bonds respectively. The bond appearing at $3,430 \mathrm{~cm}^{-1}$ is assigned for O-H stretch of alcohol/phenol. FTIR spectroscopic analysis illustrated that culture supernatant of Bacillus sp. HAI4 has the ability to perform dual functions of reduction and stabilization of AgNPs. When the metal NPs produced in the solution, they must be stabilized against the Van der Waals forces of attraction which may otherwise lead to coagulation (Fig. 6). 


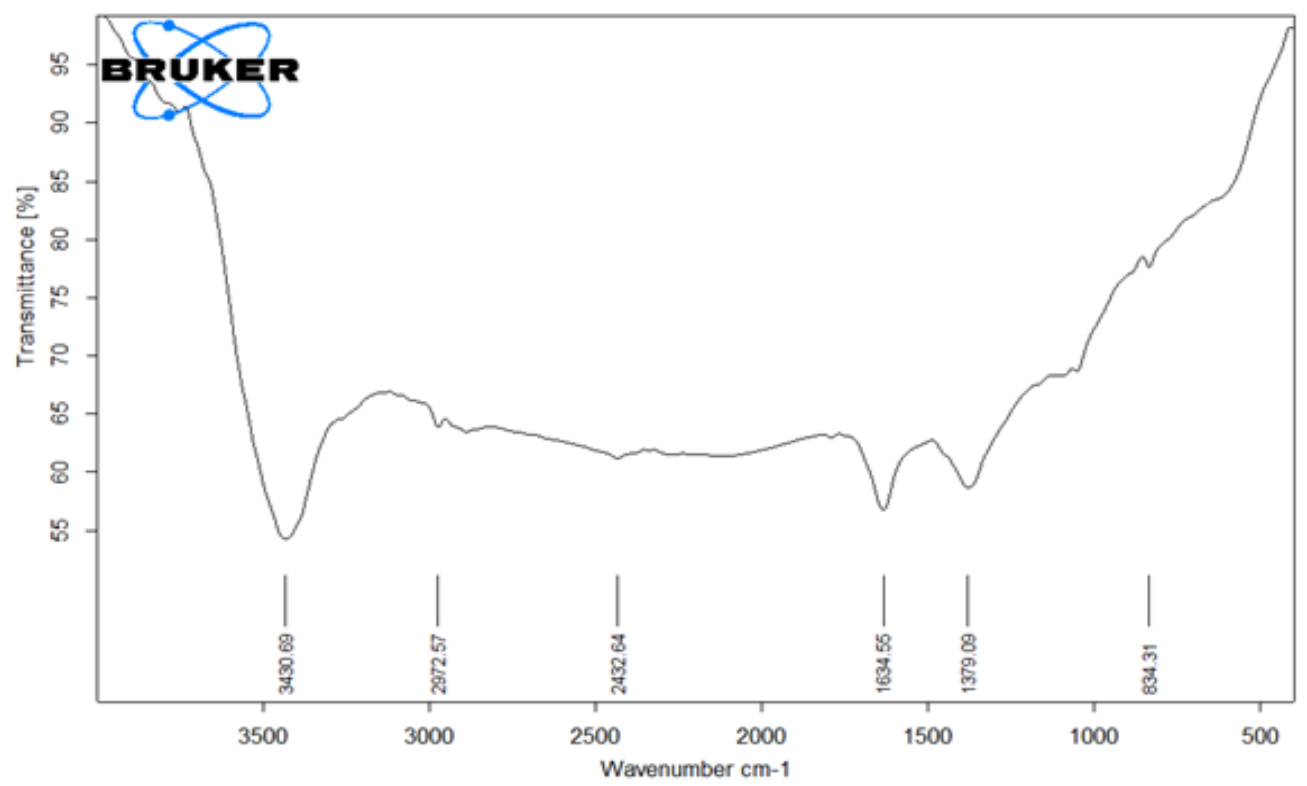

Fig. 6: FTIR spectra of AgNPs.

\section{Antibacterial activity}

Results of bactericidal effect of the nanoparticles on the pathogen bacteria are showed in table 4 . In order to evaluating of antibacterial effects, maximum zone of inhibition, two important multidrug resistant pathogenesis bacteria, Escherichia coli ATCC 25922 and Staphylococcus aureus ATCC 43300 were used (Feng et al., 2000). Three levels of Ag NPs concentrations had antibacterial effect on these bacteria $(1,1$ and $0.5 \mathrm{~cm}$ for E.coli and $1,1.1$ and 1.1 for S.aureus) but there was no significant difference between three levels (Fig 7).

Table 4: Antibacterial activity of AgNPs against E.coli ATCC 25922 and S.aureus ATCC 43300.

\begin{tabular}{lccc}
\hline & \multicolumn{3}{c}{ Zone of inhibition $(\mathbf{m m})$} \\
\hline Micro organisms & $\mathbf{0 . 1 M}$ & $\mathbf{0 . 0 1 M}$ & $\mathbf{0 . 0 0 1 M}$ \\
\hline Escherichia coli ATCC 25922 & 1 & 1 & 0.5 \\
Staphylococcus aureus ATCC 43300 & 1 & 1.1 & 1.1 \\
\hline
\end{tabular}

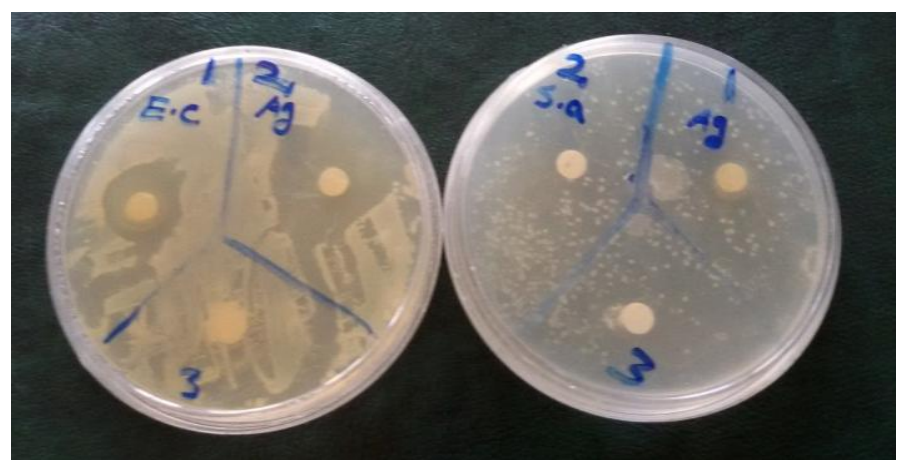

Fig. 7: Bactericidal activity of silver nanoparticles on E.coli ATCC 25922 (Left) and S.aureus ATCC 43300 (Right).
Antibacterial activity of Ag NPs is not merely resulted from their release of metal ions but also can be related to their morphology, specifically their small size and higher surface area to volume ratio. In this case, Guzman et al reported antibacterial activity of Ag NPs has been reported on the same bacterial strains (Guzman et al., 2012).

\section{CONCLUSION}

There are many studies about biosynthesis of NPs by plant, fungi, and bacteria. In this work, silver nanoparticles synthesized by Bacillus sp. HAI4 extracellulary. Stabilizing of Ag NPs was possible without using any capping agents which are toxic.

This investigation shows major effect of $\mathrm{AgNO}_{3}$ concentration on productivity of cell-free supernatant $(12.641 \%$ as Expected result at optimum condition). This performance is very important in scale-up production of NPs synthesis. Also, these NPs have antibacterial effect that can be usable in medicinal aspect for fighting against prominent pathogen bacteria E.coli ATCC 25922 and S.aureus ATCC 43300. Sondi and Salopek-Sondi (2004) reported antimicrobial effect of silver nanoparticles on $E$. coli as a model of gram-negative bacteria. In total, this study presents simple, low expensive, eco-friendly and high productivity in fabrication of Ag NPs.

\section{ACKNOWLEDGMENTS}

The authors wish to appreciate Razi University for providing necessary facilities to carry out this work.

\section{Financial support and sponsorship: Nil.}

Conflict of Interests: There are no conflicts of interest. 


\section{REFERENCES}

Abd ERRES, Sobhy EES, Mohamed EE. Extracellular Biosynthesis of Silver Nanoparticles Using Escherichia coli ATCC 8739, Bacillus subtilis ATCC 6633, and Streptococcus thermophilus ESh1 and Their Antimicrobial Activities. ISRN Nanotechnology, 2011; 20: 1-8.

Bhainsa KC, D'Souza SF. Extracellular biosynthesis of silver nanoparticles using the fungus Aspergillus fumigatus. Coll Sur B, 2006; 47: 160-164.

Blanc DS, Carrara P, Zanetti G, Francioli P. Water disinfection with ozone, copper and silver ions, and temperature increase to control Legionella: seven years of experience in a university teaching hospital. J Hosp Infect, 2005; 60: 69-72.

Faghri Zonooz N, Salouti M. Extracellular biosynthesis of silver nanoparticles using cell filtrate of Streptomyces sp. ERI-3. Scientia Iranica F, 2011; 18 (6): 1631-1635.

Feng QL, Wu J, Chen GQ, Cui FZ, Kim TN, Kim JO. A mechanistic study of the antibacterial effect of silver ions on Escherichia coli and Staphylococcus aureus. J Biomed Mater Res, 2000; 52: 662-668.

Ganesh Babu MM, Gunasekaran P. Production and structural characterization of crystalline silver nanoparticles from Bacillus cereus isolate. Coll Surf B, 2009; 74:191-195.

Guzman M, Dille j, Godet S. Synthesis and antibacterial activity of silver nanoparticles against gram-positive and gram-negative bacteria. Nanomed, 2012; 8: 37- 45.

Jain P, Pradeep T. Potential of silver nanoparticle-coated polyurethane foam as an antibacterial water filter. Biotechnol Bioeng, 2005; 90: 59-63.

Kalishwaralal K, Deepak V, Pandian SRK, Kottaisamy M, BarathManiKanth S, Kartikeyan B, Gurunathan S. Biosynthesis of silver and gold nanoparticles using Brevibacterium casei . Coll Surf B, 2010; 77: 257-262.

Lin YE, Vidic RD, Stout JE, Mc Cartney CA, Yu VL. Inactivation of Mycobacterium avium by copper and silver ions. Water Res, 1998; 32: 1997-2000.

Lin YE, Vidic RD, Stout JE, Yu VL. Individual and combined effects of copper and silver ions on inactivation of Legionella pneumophila. Water Res, 1996; 30:1905-13.

Monika A, Romuald S, Joanna K, Grazyna BP. Accumulation of Platinum Nanoparticles by Sinapis alba and Lepidium sativum Plants. Water Air Soil Poll, 2015; 226: 1-7.

Ramyadevi J, Jeyasubramanian K, Marikani A, Rajakumar G, Rahuman AA. Synthesis and antimicrobial activity of copper nanoparticles. Mater Lett, 2012; 114-116.
Saifuddin N, Wong CW, NurYasumira AA. Rapid biosynthesis of silver nanoparticles using culture supernatant of bacteria with microwave irradiation. J Chem, 2009; 6: 61-70.

Shahverdi AR, Minaeian S, Shahverdi HR, Jamalifar H, Nohi AA. Rapid synthesis of silver nanoparticles using culture supernatants of enterobacteria: a Novel Biological Approach. Process Biochem, 2007; 42: 919-923.

Sondi I, Salopek-Sondi, B. Silver nanoparticles as antimicrobial agent: a case study on E. coli as a model Gram-negative bacteria. J Colloid Interface Sci, 2004; 275: 177-182.

Taran M, Sisakhtnezhad S, Azin T. Biological removal of nickel (II) by Bacillus sp. KL1 in different conditions: optimization by Taguchi statistical approach. Pol J Chem Technol, 2015; 17: 10-15.

Varshney R, Bhaduria S, Mulayam S. Gaur. A Review: Biological Synthesis of Silver and Copper Nanoparticles. Nano Biomed Eng. 2012; 4(2): 99-106.

Vaseashta A, Dimova-Malinovska D. Nanostructured and nanoscale devices, sensors and detectors. Sci Technol Adv Mater, 2005; 6:312-18.

Vicky V, Mody, Rodney S, Ajay S, Hardik RM. Introduction to metallic nanoparticles. J Pharm Bioallied Sci, 2010; 2(4): 282-289.

Vidhya LD, Roshmi T, Rintu TV, Soniya EV, Jyothis M, Radhakrishnan EK. Extracellular synthesis of silver nanoparticles by the Bacillus strain CS 11 isolated from industrialized area. 3 Biotech, 2014; 4(2): 121-126.

Yoon K, Byeon JH, Park J, Hwang J. Susceptibility constants of E. coli and Bacillus subtilis to $\mathrm{Ag}$ and $\mathrm{Cu}$ nanoparticles. Sci Total Environ, 2007; 373:572-5.

\section{How to cite this article:}

Taran M, Rad M, Alavi M. Characterization of Ag nanoparticles biosynthesized by Bacillus sp. HAI4 in different conditions and their antibacterial effects. J App Pharm Sci, 2016; 6 (11): 094-099. 